Korean J. Math. 20 (2012), No. 3, pp. 353-360

\title{
GENERALIZED FIXED POINT THEOREMS IN CONE METRIC SPACES
}

\author{
Seung Hyun Kim And Byung Soo LeE*
}

\begin{abstract}
In this paper we introduce the property $(C)$, which is a cone metric extension of the usual metric property $(E, A)$ and consider fixed point theorems for generalized contractive mappings under suitable conditions in cone metric spaces without normal cones.
\end{abstract}

\section{Introduction and Preliminaries}

In 2007, Huang and Zhang [1] introduced a cone metric space with a cone metric generalizing the usual metric space by replacing the real numbers with Banach spaces ordered by the cone. They considered some fixed point theorems for contractive mappings in cone metric spaces with normal cones. Since then, the fixed point theory for mappings in cone metric spaces with normal cones has become a subject of interest in nonlinear analysis [1-6].

In 2002, Aamri and Moutawakil [8] introduced a property $(E, A)$ for self mappings and obtained some fixed point theorems for such mappings under strict contractive conditions. The class of mappings satisfying property $(E, A)$ contains the class of noncompatible mappings. The property $(E, A)$ is very useful in the study of fixed point theorems of nonexpansive mappings, see [9].

Inspired and encouraged by the previous works, in this paper we the authors introduce the property $(C)$, which is a cone metric extension of the usual metric property $(E, A)$ and consider some fixed point theorems

Received August 12, 2012. Revised September 15, 2012. Accepted September 16, 2012.

2010 Mathematics Subject Classification: 47H10, 54C60.

Key words and phrases: cone metric space, contractive mapping, quasi weak contractive mapping, the property $(E, A)$, the property $(C)$, fixed point, common fixed point, coincidence point.

${ }^{*}$ Corresponding author. 
for generalized contractive mappings under suitable conditions in cone metric spaces without normal cones.

A nonempty subset $P$ of a real Banach space $E$ is called a (pointed) cone if and only if

(P1) $P$ is closed, $P \neq \emptyset, P \neq\{0\}$;

(P2) $a, b \in \mathbb{R}$ with $a, b \geq 0, x, y \in P \Rightarrow a x+b y \in P$;

(P3) $x \in P$ and $-x \in P \Rightarrow x=0$.

Let $P \subset E$ be a cone; we define a partial ordering ' $\preceq$ ' with respect to $P$ as follows; for $x, y \in E$, we say that $x \preceq y$ if and only if $y-x \in P$, $x \ll y$ if and only if $y-x \in \operatorname{int} P$, where int $P$ denotes the interior of $P$, $x \prec y$ if and only if $x \preceq y$ and $x \neq y$.

Definition 1.1. [1] Let $M$ be a nonempty set. Suppose that a mapping $d: M \times M \rightarrow(E, P)$ satisfies the following;

(d1) $0 \preceq d(x, y)$ for all $x, y \in M$ and $d(x, y)=0$ if and only if $x=y$;

(d2) $d(x, y)=d(y, x)$ for all $x, y \in M$;

(d3) $d(x, y) \preceq d(x, z)+d(y, z)$ for all $x, y, z \in M$.

Then $d$ is called a cone metric on $M$, and $(M, d)$ is called a cone metric space.

The following definitions and lemmas are considered in a cone metric space $(M, d)$.

Definition 1.2. [1] Let $\left\{x_{n}\right\}$ be a sequence in $M$ and $x \in M$. If for every $c \in$ int $P$, there is a natural number $N$ such that for all $n>N$, $d\left(x_{n}, x\right) \ll c$, then we say that $\left\{x_{n}\right\}$ converges to $x$ with respect to $P$ and denote as $\lim _{n \rightarrow \infty} x_{n}=x$.

Lemma 1.1. [1] Let $P$ be a cone. Let $\left\{x_{n}\right\}$ and $\left\{y_{n}\right\}$ be sequences in M. Then;

(i) $\left\{x_{n}\right\}$ converges to $x$ with respect to $P$ if and only if $d\left(x_{n}, x\right) \rightarrow 0$ as $n \rightarrow \infty$;

(ii) If $x_{n} \rightarrow x$ and $y_{n} \rightarrow y$ as $n \rightarrow \infty$ with respect to $P$, then $d\left(x_{n}, y_{n}\right) \rightarrow$ $d(x, y)$ as $n \rightarrow \infty$.

(iii) If $x_{n} \rightarrow x$ and $y_{n} \rightarrow y$ as $n \rightarrow \infty$ with respect to $P$ and $x_{n} \preceq y_{n}$ for all $n \in \mathbb{N}$, then $x \preceq y$.

Definition 1.3. Two mappings $S, T: M \rightarrow M$ are weakly compatible if $S T x=T S x$ whenever $S x=T x$. 


\section{Fixed point theorems in cone metric spaces}

Now, we introduce some property in cone metric spaces, which can be helpful to check the relationship of a limit of sequence converging to some point and a limit of the image sequence converging to some point.

DeFinition 2.1. Let $M$ be a nonempty set with a cone metric $d$ : $M \times M \rightarrow(E, P)$. A mapping $T: M \rightarrow M$ is said to satisfy the property $(C)$ if there is a sequence $\left\{x_{n}\right\}$ in $M$ such that

$$
\lim _{n \rightarrow \infty} d\left(x_{n}, z\right)=0=\lim _{n \rightarrow \infty} d\left(T x_{n}, z\right) \text { for some } z \in M \text {. }
$$

ExAmple 2.1. Let $M=[0,1], E=\mathbb{R}^{2}$ be a Banach space with the standard norm, $P=\{(x, y) \in E ; x, y \geq 0\}$ be a cone and let $d$ : $M \times M \rightarrow E$ be a mapping of the form $d(x, y)=\left(|x-y|, \frac{1}{2}|x-y|\right)$. Then the pair $(M, d)$ is a cone metric space. Define a mapping $T: M \rightarrow M$ by $T x=\frac{x}{2}$. Consider a sequence $\left\{x_{n}\right\}=\left\{\frac{1}{n}\right\}$, for all $n \in \mathbb{N}$. Then

$$
\lim _{n \rightarrow \infty} d\left(x_{n}, 0\right)=0=\lim _{n \rightarrow \infty} d\left(T x_{n}, 0\right) .
$$

Hence, $T$ satisfies the property $(C)$.

REMARK 2.1. The property $(E, A)$ considered in [8] is a usual metric case of the property $(C)$.

We introduce generalized $(\psi, \varphi)$-weak contractive mapping in cone metric spaces.

DeFinition 2.2. Let $M$ be a nonempty set with a cone metric $d$ : $M \times M \rightarrow(E, P)$. A mapping $T: M \rightarrow M$ is said to be generalized $(\psi, \varphi)$-weak contractive if for each $x, y \in M$,

$$
\psi(d(T x, T y)) \preceq \psi(d(x, y))-\varphi(d(x, y)),
$$

where $\psi, \varphi \in \Phi=\{\varphi ; \varphi: P \rightarrow P$ a continuous mapping satisfying $\varphi(t)=$ 0 iff $t=0\}$.

ExAmple 2.2. Let $M, E, P$ and $d$ be the same as in Example 2.1. Define mappings $\psi, \varphi: P \rightarrow P$ by $\psi((x, y))=\left(\frac{x}{2}, y\right), \varphi((x, y))=$ 
$\left(\frac{1}{4} x^{2}, y^{2}\right)$. Define a mapping $T: M \rightarrow M$ by $T x=x-\frac{1}{2} x^{2}$. Without loss of generality, we assume that $x>y$.

$$
\begin{aligned}
\psi(d(T x, T y)) & =\psi\left(\left(\left|x-\frac{1}{2} x^{2}-y+\frac{1}{2} y^{2}\right|, \frac{1}{2}\left|x-\frac{1}{2} x^{2}-y+\frac{1}{2} y^{2}\right|\right)\right) \\
& =\psi\left(\left((x-y)-\frac{1}{2}(x-y)(x+y), \frac{1}{2}\left\{(x-y)-\frac{1}{2}(x-y)(x+y)\right\}\right)\right) \\
& =\left(\frac{1}{2}\left\{(x-y)-\frac{1}{2}(x-y)(x+y)\right\}, \frac{1}{2}\left\{(x-y)-\frac{1}{2}(x-y)(x+y)\right\}\right) \\
& \preceq\left(\frac{1}{2}\left\{(x-y)-\frac{1}{2}(x-y)^{2}\right\}, \frac{1}{2}\left\{(x-y)-\frac{1}{2}(x-y)^{2}\right\}\right) \\
& =\left(\frac{1}{2}(x-y), \frac{1}{2}(x-y)\right)-\left(\frac{1}{4}(x-y)^{2}, \frac{1}{4}(x-y)^{2}\right) \\
& =\psi\left(\left(x-y, \frac{1}{2}(x-y)\right)\right)-\varphi\left(\left(x-y, \frac{1}{2}(x-y)\right)\right) \\
& =\psi(d(x, y))-\varphi(d(x, y))
\end{aligned}
$$

Hence, $T$ is a $(\psi, \varphi)$-weak contraction.

REMARK 2.2. Generalized $(\psi, \varphi)$-weak contraction is a cone metric extension of $(\psi, \varphi)$-weak contraction considered in [7].

Now, we introduce quasi-weak contractive mapping in cone metric spaces.

Definition 2.3. Let $M$ be a nonempty set with a cone metric $d$ : $M \times M \rightarrow(E, P)$. A mapping $T: M \rightarrow M$ is said to be quasi weak contractive if for each $x, y \in M$,

$$
\psi(d(T x, T y)) \preceq \psi\left(M_{T}(x, y)\right)-\varphi\left(M_{T}(x, y)\right), \quad \text { for } \quad \psi, \varphi \in \Phi
$$

provided that

$$
M_{T}(x, y):=\max \{d(x, y), d(x, T x), d(y, T y), d(x, T y), d(y, T x)\} .
$$

REMARK 2.3. Quasi-weak contraction is an extension of generalized $(\psi, \varphi)$-weak contraction.

THEOREM 2.1. Let $M$ be a nonempty set with a cone metric $d$ : $M \times M \rightarrow(E, P)$ and $T: M \rightarrow M$ a quasi-weak contraction satisfying the property $(C)$. Then $T$ has a unique fixed point.

Proof. Let $\left\{x_{n}\right\}$ be a sequence in $M$ satisfying

$$
\lim _{n \rightarrow \infty} d\left(x_{n}, z\right)=0=\lim _{n \rightarrow \infty} d\left(T x_{n}, z\right) \text { for some } z \in M .
$$


Since $T$ is a quasi-weak contraction,

$$
\psi\left(d\left(T z, T x_{n}\right)\right) \preceq \psi\left(M_{T}\left(z, x_{n}\right)\right)-\varphi\left(M_{T}\left(z, x_{n}\right)\right),
$$

for $\psi, \varphi \in \Phi$. From (2.1), we have

$$
\begin{aligned}
\lim _{n \rightarrow \infty} & M_{T}\left(z, x_{n}\right) \\
& =\lim _{n \rightarrow \infty} \max \left\{d\left(z, x_{n}\right), d(z, T z), d\left(x_{n}, T x_{n}\right), d\left(z, T x_{n}\right), d\left(x_{n}, T z\right)\right\} \\
& =\max \{d(z, z), d(z, T z), d(z, z), d(z, z), d(z, T z)\} \\
& =d(z, T z) .
\end{aligned}
$$

From (2.2) and (2.3),

$$
\begin{aligned}
& \lim _{n \rightarrow \infty} \psi\left(d\left(T z, T x_{n}\right)\right) \preceq \lim _{n \rightarrow \infty}\left(\psi\left(M_{T}\left(z, x_{n}\right)\right)-\varphi\left(M_{T}\left(z, x_{n}\right)\right)\right) \\
& \quad \Rightarrow \psi(d(T z, z)) \preceq \psi(d(z, T z))-\varphi(d(z, T z)) \\
& \quad \Rightarrow d(T z, z)=0 .
\end{aligned}
$$

Thus $\mathrm{z}$ is a fixed point of $\mathrm{T}$. To prove the uniqueness, suppose that $T$ has two distinct fixed points $y$ and $z$ in $M$, then

$$
\begin{aligned}
M_{T}(y, z) & =\max \{d(y, z), d(y, T y), d(z, T z), d(y, T z), d(z, T y)\} \\
& =\max \{d(y, z), d(y, y), d(z, z), d(y, z), d(z, y)\} \\
& =d(y, z) .
\end{aligned}
$$

Since $T$ is a $(\psi, \varphi)$-weak contraction,

$$
\begin{aligned}
\psi(d(y, z)) & =\psi(d(T y, T z)) \preceq \psi\left(M_{T}(y, z)\right)-\varphi\left(M_{T}(y, z)\right) \\
& =\psi(d(y, z))-\varphi(d(y, z)) \\
& \Rightarrow \varphi(d(y, z))=0,
\end{aligned}
$$

which implies the unique existence of fixed point of $T$.

If $M_{T}(x, y)=d(x, y)$, then we have the following theorem from Theorem 2.1 as a corollary.

TheOrem 2.2. Let $M$ be a nonempty set with a cone metric $d$ : $M \times M \rightarrow(E, P)$ and $T: M \rightarrow M$ a generalized $(\psi, \varphi)$-weak contraction satisfying the property $(C)$. Then $T$ has a unique fixed point.

By putting $\psi(t)=t$ and $\varphi(t)=0$ in Theorem 2.2, we obtain the following fixed point theorem for a nonexpansive mapping in cone metric spaces. 
THEOREM 2.3. Let $M$ be a nonempty set with a cone metric $d$ : $M \times M \rightarrow(E, P)$ and $T: M \rightarrow M$ a mapping satisfying the property $(C)$ such that $d(T x, T y) \preceq d(x, y)$ for all $x, y \in M$. Then $T$ has a unique fixed point.

\section{Common fixed point theorems in cone metric spaces}

In this section, we obtain a coincidence and common fixed point theorem in cone metric spaces.

Definition 3.1. Let $M$ be a nonempty set with a cone metric $d$ : $M \times M \rightarrow(E, P)$. Two mappings $S, T: M \rightarrow M$ are said to satisfy the property $(C)$ if there is a sequence $\left\{x_{n}\right\}$ in $M$ such that

$$
\lim _{n \rightarrow \infty} d\left(S x_{n}, z\right)=0=\lim _{n \rightarrow \infty} d\left(T x_{n}, z\right) \text { for some } z \in M \text {. }
$$

Example 3.1. Let $M, E, P$ and $d$ be the same as in Example 2.1. Define two mappings $S, T: M \rightarrow M$ by $T x=\frac{x}{2}$ and $S x=\frac{x^{2}}{2}$. Consider a sequence $\left\{x_{n}\right\}=\left\{\frac{1}{n}\right\}$, for all $n \in \mathbb{N}$. Then

$$
\lim _{n \rightarrow \infty} d\left(S x_{n}, 0\right)=0=\lim _{n \rightarrow \infty} d\left(T x_{n}, 0\right) .
$$

Hence, $S$ and $T$ satisfy the property $(C)$.

THEOREM 3.1. Let $M$ be a nonempty set with a cone metric $d$ : $M \times M \rightarrow(E, P)$ and $S, T: M \rightarrow M$ be mappings satisfying the property $(C), S$ is onto, and for each $x, y \in M$,

$$
\psi(d(T x, T y)) \preceq \psi(d(S x, S y))-\varphi(d(S x, S y))
$$

for $\psi, \varphi \in \Phi$. Then $S$ and $T$ have a coincidence point in $M$. Moreover, if $S$ and $T$ are weakly compatible, then $S$ and $T$ have a unique common fixed point.

Proof. Let $\left\{x_{n}\right\}$ be a sequence in $M$ satisfying

$$
\lim _{n \rightarrow \infty} d\left(S x_{n}, z\right)=0=\lim _{n \rightarrow \infty} d\left(T x_{n}, z\right) \text { for some } z \in M .
$$

Take $a \in M$ such that $z=S a$, then

$$
\lim _{n \rightarrow \infty} d\left(S x_{n}, S a\right)=0=\lim _{n \rightarrow \infty} d\left(T x_{n}, S a\right) \text { for some } z \in M .
$$

Since

$$
\psi\left(d\left(T a, T x_{n}\right)\right) \preceq \psi\left(d\left(S a, S x_{n}\right)\right)-\varphi\left(d\left(S a, S x_{n}\right)\right)
$$


we have

$$
\begin{aligned}
& \lim _{n \rightarrow \infty} \psi\left(d\left(T a, T x_{n}\right)\right) \preceq \lim _{n \rightarrow \infty}\left(\psi\left(d\left(S a, S x_{n}\right)\right)-\varphi\left(\left(S a, S x_{n}\right)\right)\right) \\
& \quad \Rightarrow \psi(d(T a, S a)) \preceq \psi(d(S a, S a))-\varphi(d(S a, S a)) \\
& \quad \Rightarrow d(T a, S a)=0
\end{aligned}
$$

Since $S$ and $T$ are weakly compatible, $S S a=S T a=T S a=T T a$.

Now, we show that $z=T a$ is a common fixed point of $S$ and $T$. We have

$$
\begin{aligned}
\psi(d(T a, T T a)) & \preceq \psi(d(S a, S T a))-\varphi(d(S a, S T a)) \\
& =\psi(d(T a, T T a))-\varphi(d(T a, T T a)) \\
& \Rightarrow T a=T T a .
\end{aligned}
$$

Hence $T T a=S T a=T a=z$. To prove the uniqueness, suppose that $S$ and $T$ have two distinct fixed points $y=S y=T y$ and $z=S z=T z$ in $M$, then

$$
\begin{aligned}
\psi(d(T z, T y)) & \preceq \psi(d(S z, S y))-\varphi(d(S z, S y)) \\
& =\psi(d(T z, T y))-\varphi(d(T z, T y)) \\
& \Rightarrow \varphi(d(T z, T y))=0 .
\end{aligned}
$$

REMARK 3.1. In [11], the common fixed point results are proved under the assumption that the cone is regular. However, in the proof of Theorem 3.1, we do not use the assumption that the cone is regular.

By putting $\psi(t)=t$ and $\varphi(t)=0$ in Theorem 3.1, we obtain the following common fixed point theorem.

Theorem 3.2. Let $M$ be a nonempty set with a cone metric $d$ : $M \times M \rightarrow(E, P)$ and $S, T: M \rightarrow M$ be mappings satisfying the property $(C), S$ is onto, and for each $x, y \in M$,

$$
d(T x, T y) \preceq d(S x, S y) .
$$

Then $S$ and $T$ have a coincidence point in $M$. Moreover, if $S$ and $T$ are weakly compatible, then $S$ and $T$ have a unique common fixed point.

The following theorem in [10] is a corollary of Theorem 3.2.

Theorem 3.3. Let $(X, d)$ be a cone metric space, and $P$ a normal cone with normal constant $K$. Suppose mappings $S, T: M \rightarrow M$ satisfy

$$
d(T x, T y) \preceq k d(S x, S y), \text { for all } x, y \in M,
$$


where $k \in[0,1)$ is a constant. If the range of $S$ contains the range of $T$ and $S(M)$ is a complete subspace of $M$, then $T$ and $S$ have a unique point of coincidence in $M$. Moreover, if $S$ and $T$ are weakly compatible, then $S$ and $T$ have a unique common fixed point.

\section{References}

[1] L.G. Huang and X. Zhang, Cone metric spaces and fixed point theorems of contractive mappings, J. Math. Anal. Appl. 332 (2007), 1468-1476.

[2] D. Wardowski, On set-valued contractions of Nadler type in cone metric spaces, Appl. Math. Lett. 24 (2011), 275-278.

[3] I. Altun, B. Damjanović and D. Djorić, Fixed point and common fixed point theorems on ordered cone metric spaces, Appl. Math. Lett. 23 (2010), 310-316.

[4] D. Ilić and V. Rakočević, Quasi-contraction on a cone metric space, Appl. Math. Lett. 22 (2009), 728-731.

[5] D. Klim and D. Wardowski, Dynamic processes and fixed points of set-valued nonlinear contraction in cone metric spaces, Nonlinear Anal. 71 (2009), 51705175 .

[6] B.C. Choudhury and N. Metiya, Fixed points of weak contractions in cone metric spaces, Nonlinear Anal. 72 (2010), 1589-1593.

[7] P.N. Dutta and B.S. Choudhury, A generalisation of contraction principle in metric spaces, Fixed Point Theory Appl. 2008 (2008), Article ID 406386, 8pages.

[8] M. Aamri and D.E. Moutawakil, Some new common fixed point theorems under strict contractive conditions, J. Math. Anal. Appl. 270 (2002), 181-188.

[9] R.P. Pant, Fixed points of nonexpansive mappings and a generalized notion of compactness, Bull. Calcutta Math. Soc. 99 (1) (2007), 45-52.

[10] M. Abbas and G. Jungck, Common fixed point results for noncommuting mappings without continuity in cone metric spaces, J. Math. Anal. Appl. 341 (2008), 416-420.

[11] H.R. Zhao, B.S. Lee and N.J. Huang, Common fixed points for generalized weakly contractive mappings in cone metric spaces, Nonlinear Anal. Forum 16 (2011), $77-84$.

Department of Mathematics

Kyungsung University

Busan 608-736, Korea

E-mail: jiny0610@hotmail.com

Department of Mathematics

Kyungsung University

Busan 608-736, Korea

E-mail: bslee@ks.ac.kr 